\title{
Disulfiram, a Re-positioned Aldehyde Dehydrogenase Inhibitor, Enhances Radiosensitivity of Human Glioblastoma Cells In Vitro
}

\author{
Hyeon Kang Koh, $\mathrm{MD}^{1,2}$ \\ Soo Yeon Seo, BS ${ }^{3}$ \\ Jin Ho Kim, MD, PhD 2,3 \\ Hak Jae Kim, MD, PhD2,3 \\ Eui Kyu Chie, MD, PhD23, \\ Seung-Ki Kim, MD, PhD ${ }^{4}$ \\ II Han Kim, MD, PhD2,3
}

\begin{abstract}
Purpose
Glioblastoma, the most common brain tumor in adults, has poor prognosis. The purpose of this study was to determine the effect of disulfiram (DSF), an aldehyde dehydrogenase inhibitor, on in vitro radiosensitivity of glioblastoma cells with different methylation status of $0^{6}$-methylguanine-DNA methyltransferase (MGMT) promoter and the underlying mechanism of such effect.
\end{abstract}

\section{Materials and Methods}

Five human glioblastoma cells (U138MG, T98G, U251MG, U87MG, and U373MG) and one normal human astrocyte (NHA) cell were cultured and treated with DSF or $6 \mathrm{MV} \mathrm{X-rays}(0,2$, 4,6 , and 8 Gy). For combined treatment, cells were treated with DSF before irradiation. Surviving fractions fit from cell survival based on colony forming ability. Apoptosis, DNA damage repair, and cell cycle distribution were assayed by western blot for cleaved caspase-3, yH2AX staining, and flow cytometry, respectively.

\section{Results}

DSF induced radiosensitization in most of the glioblastoma cells, especially, in the cells with radioresistance as wildtype unmethylated promoter (MGMT-wt), but did not in normal NHA cell. DSF augmented or induced cleavage of caspase-3 in all cells after irradiation. DSF inhibited repair of radiation-induced DNA damage in MGMT-wt cells, but not in cells with methylated MGMT promoter. DSF abrogated radiation-induced G2/M arrest in T98G and U251MG cells.

\section{Conclusion}

Radiosensitivity of glioblastoma cells were preferentially enhanced by pre-irradiation DSF treatment compared to normal cell, especially radioresistant cells such as MGMT-wt cells. Induction of apoptosis or inhibition of DNA damage repair may underlie DSF-induced radiosensitization. Clinical benefit of combining DSF with radiotherapy should be investigated in the future.
Correspondence: Il Han Kim, MD, PhD Department of Radiation Oncology,

Seoul National University College of Medicine, 101 Daehak-ro, Jongno-gu, Seoul 03080, Korea

Tel: 82-2-2072-2528

Fax: 82-2-765-3317

E-mail: ihkim@snu.ac.kr

Received April 26, 2018

Accepted August 10, 2018

Published Online August 13, 2018
Key words

Disulfiram, Glioblastoma, MGMT, Radiosensitivity,

DNA damage repair, Apoptosis

\section{Introduction}

Glioblastoma (GBL), the most common brain tumor in adults, has very poor prognosis. Even with the current standard therapy of maximal resection followed by radiotherapy concurrently with temozolomide (TMZ) and TMZ maintenance, the median survival (MS) was 14.6 months in overall patients and less than 24 months in patients with favorable parameters [1,2].

As an anti-mutagenic DNA repair protein, $\mathrm{O}^{6}$-methylguanine-DNA methyltransferase (MGMT) interferes with the anti-cancer effect of TMZ by removing methyl group attached to DNA [3]. Survival of GBL patients depends on the status of MGMT promotor methylation. Those with methylated MGMT promoter (MGMT-meth) have better survival 
than those with wildtype unmethylated MGMT promoter (MGMT-wt) [3,4]. A novel recursive partitioning analysis category has been formulated using MGMT promotor methylation and isocitrate dehydrogenase 1 (IDH1) mutation in addition to traditional demographic parameters for newly diagnosed GBL in TMZ era, emphasizing the status of MGMT promotor methylation as the first partitioning generator [5]. MS was 32.8 months for MGMT-meth and 18.7 months MGMT-wt GBL, respectively $(\mathrm{p}<0.001)$.

Since the establishment of standard radiochemotherapy with TMZ around 2006, several attempts have been made to further improve treatment outcomes of GBL, especially GBL with MGMT-wt. Bevacizumab, a monoclonal antibody that binds vascular endothelial growth factor, has failed to provide survival benefit in large phase III studies of AVAglio and RTOG 0825 [6,7]. Although addition of tumor-treating field (TTF) to maintenance TMZ has improved MS from 16.6 months to 19.4 months, the relationship between methylation status of MGMT and treatment outcome remains unclear [8].

Disulfiram (DSF), an aldehyde dehydrogenase (ALDH) inhibitor, has been used for alcoholics since its approval by the United States Food and Drug Administration in 1948 [9]. It produces severe hangover due to accumulation of acetaldehyde after consuming alcohol, and was tolerable without severe toxicities if a person refrains from alcohol consumption [9]. DSF has recently been proposed as a potential repositioned anti-cancer drug [10] or an ALDH targeting agent for cancer stem cells [11]. It induces diverse biological effects such as inactivation of nuclear factor $\mathrm{\kappa B}$, accumulation of ubiquitination protein, amplification of reactive oxygen species, inhibition of proteosomes, and suppression of DNA methyltransferases [12]. DSF causes MGMT degradation $[13,14]$. It has synergistic effects with TMZ on GBL cells [15]. DSF can also potentiate radiation cytotoxicity in atypical teratoid / rhabdoid tumor (AT/RT) cells [11,16], neuroblastoma cells [17], and pancreatic cancer cells [18]. However, the combined effect of DSF and ionizing radiation on GBL cells has never been reported yet.

Therefore, the objective of the present study was to investigate the in vitro radiosensitizing effect of DSF on GBL cells with different status of MGMT promoter methylation and the underlying mechanism of such effect.

\section{Materials and Methods}

\section{Cell culture and drug preparation}

Five human GBL cells were used in this study. T98G and U251MG cells were obtained from the American Type Cul- ture Collection. U87MG and U373MG cells were from the Korean Cell Line Bank. U138MG cells and a normal human astrocyte (NHA) cells were provided by colleagues. U138MG, U251MG, U87MG, and NHA cells were expanded in Dulbecco's modified Eagle's medium while T98G and U373MG were cultured in RPMI 1640 medium supplemented with $10 \%$ fetal bovine serum and $1 \%$ antibiotics.

DSF was purchased from Sigma-Aldrich Chemical Co. (St. Louis, MO). It was dissolved in dimethyl sulfoxide (SigmaAldrich) to create concentrated stock solutions that were stored at $-20^{\circ} \mathrm{C}$. A stock solution was diluted in culture medium at the time of use. The $50 \%$ inhibitory concentration $\left(\mathrm{IC}_{50}\right)$ of each cell line was determined using clonogenic assay after exposing cells to increasing concentrations of DSF for 24 hours.

\section{Clonogenic assay}

Cell survival was measured using clonogenic assay in triplicates as previously reported [19]. Briefly, cells were irradiated with graded doses of $0,2,4,6$, and 8 Gy of 6 MV X-ray (Clinac 6EX, Varian Medical Systems, Palo Alto, CA). Cell survival data were fitted to a linear-quadratic (LQ) model [20]. Clonogenic assay was repeated three to four times for each cell line.

\section{Western blot for cleaved caspase-3 and MGMT}

Western blotting was undertaken as previously reported [21]. Antibodies for cleaved caspase-3 and MGMT were obtained from Cell Signaling Technology (Beverly, MA). Western blot for MGMT expression was repeated three times for all GBL cell lines treated or not treated with DSF for 24 hours. After DSF treatment for 24 hours followed by irradiation with X-ray dose of $6 \mathrm{~Gy}$, cells were subjected to western blot for cleaved capase-3 at 0, 2, 6, and 24 hours after irradiation. These processes were repeated twice.

\section{4. $\gamma \mathrm{H} 2 \mathrm{AX}$ immunocytochemistry}

Immunocytochemistry of $\gamma \mathrm{H} 2 \mathrm{AX}$ as a marker for detecting DNA damage was assayed as previously reported [19]. After exposure to DSF for 24 hours followed by irradiation with X-ray dose of $6 \mathrm{~Gy}$, cells were subjected to $\gamma \mathrm{H} 2 \mathrm{AX}$ immunocytochemistry analysis at $0,2,6$, and 24 hours after irradiation. For each treatment condition, numbers of $\gamma \mathrm{H} 2 \mathrm{AX}$ foci in 50 cells were counted. Cells with more than five foci of $\gamma \mathrm{H} 2 \mathrm{AX}$ per nucleus were considered as positive (i.e., containing radiation-induced $\gamma \mathrm{H} 2 \mathrm{AX}$ foci). The process was repeated twice for all cell lines. 


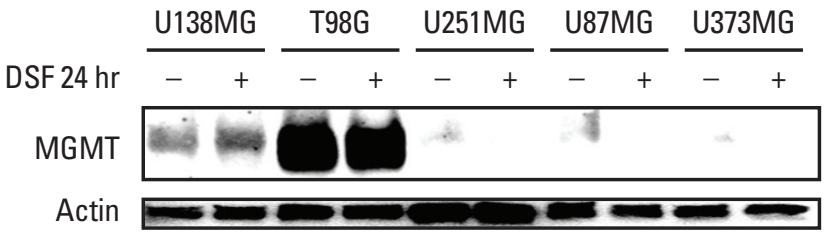

Fig. 1. Expression of $\mathrm{O}^{6}$-methylguanine-DNA methyltransferase (MGMT) protein in glioblastoma cells. U138MG and T98G cells expressed MGMT whereas U251MG, U87MG, or U373MG cells did not express MGMT based on western blots. After disulfiram (DSF) treatment for 24 hours, U138MG and T98G cells maintained expression of MGMT.

\section{Flow cytometry}

Flow cytometry was performed as previously reported [22]. After exposure to DSF for 24 hours followed by irradiation with X-ray dose of $6 \mathrm{~Gy}$, cells were subjected to flow cytometry analysis at $0,2,6$, and 24 hours after irradiation using a FACScan (Becton Dickinson, Franklin Lakes, NJ). At least $5 \times 10^{5}$ events were counted. Each procedure was performed twice for all cell lines.

\section{Statistical analysis}

Kaleidagraph ver. 3.51 (Synergy Software, Reading, PA) was applied to fit survival data of irradiated cells into LQ model. Mean values between two groups was compared using Student's t test. All statistical analyses were undertaken using SPSS ver. 18.0 (SPSS Inc., Chicago, IL).

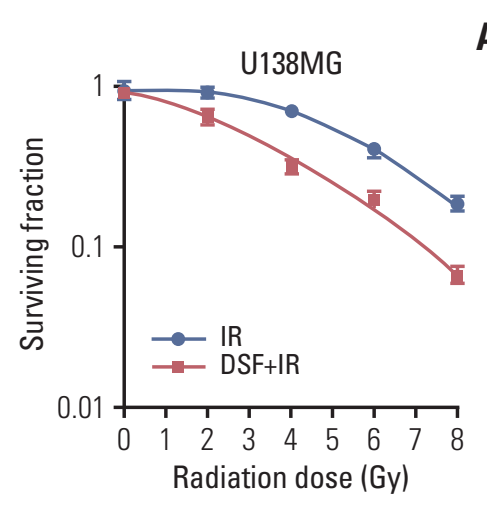

A

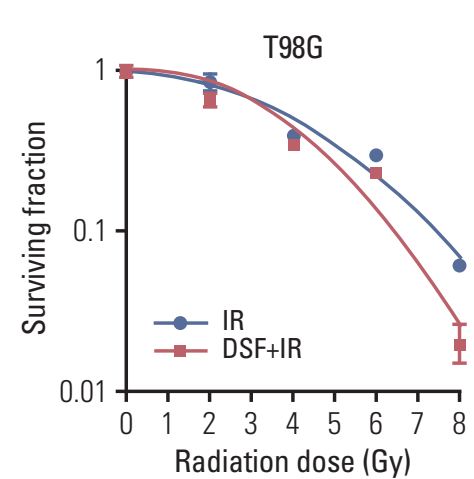

D

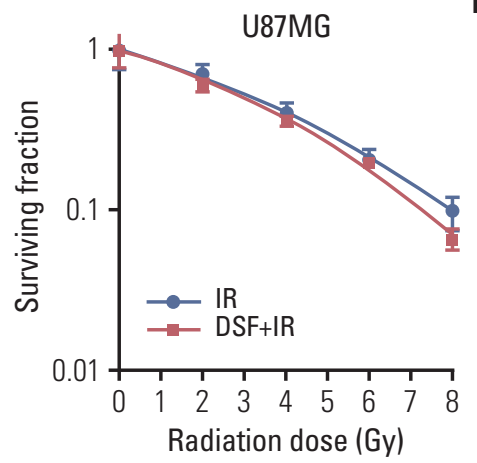

B

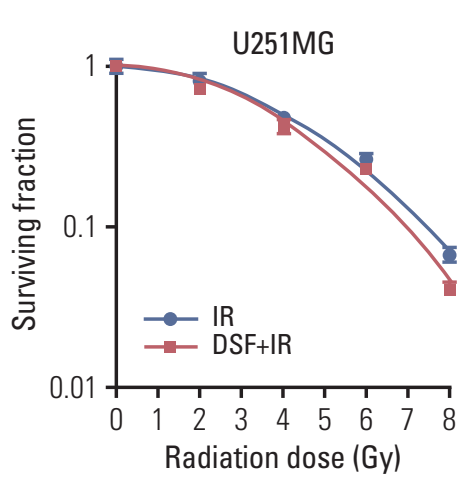

$\mathbf{E}$

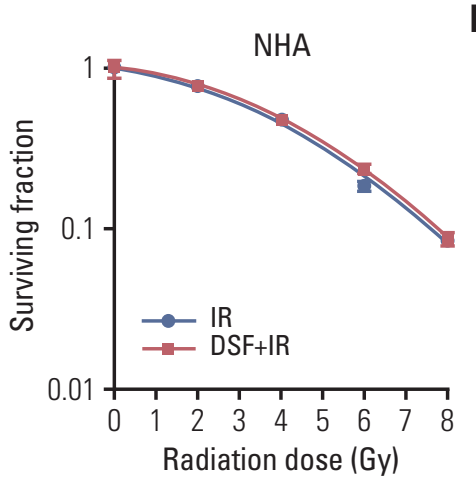

Fig. 2. (A-F) Cell survival curves fitting to a linear-quadratic model after irradiation or pre-irradiation disulfiram (DSF) treatment. Radiosensitivity of glioblastoma cells was enhanced by pre-irradiation DSF treatment except for U373MG (E) or normal human astrocyte (NHA) (F). Enhancement was especially pronounced in U138MG (A) and T98G (B) cells with wildtype unmethylated $\mathrm{O}^{6}$-methylguanine-DNA methyltransferase promoter. Each experiment was repeated at least three times with similar results. IR, irradiation. 


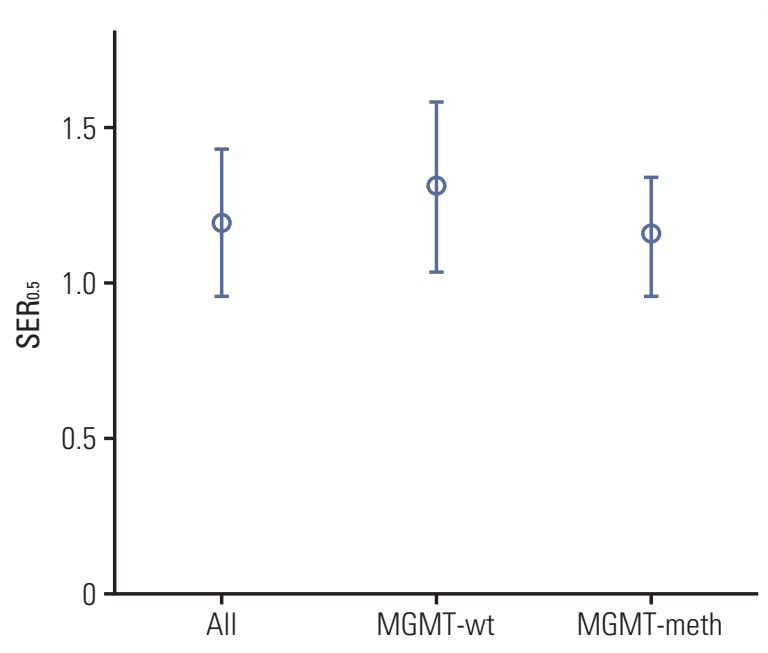

A

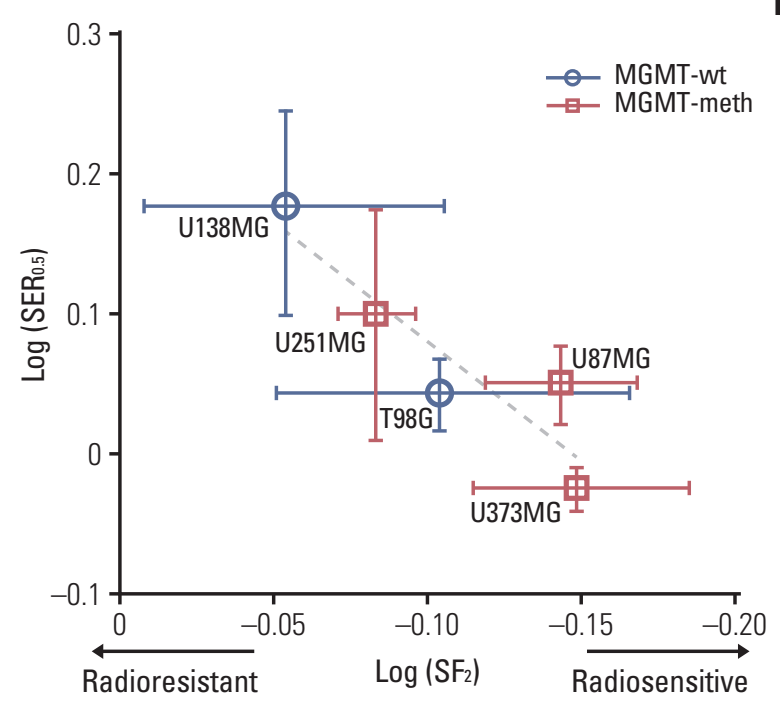

Fig. 3. Sensitizer enhancement ratio at $0.5\left(\mathrm{SER}_{0.5}\right)$. (A) Mean SER 0.5 value \pm standard deviation (SD) in all cells. Wildtype unmethylated $\mathrm{O}^{6}$-methylguanine-DNA methyltransferase (MGMT) promoter (MGMT-wt) cells and methylated MGMT promoter (MGMT-meth) cells are indicated. (B) The relationship between survival fraction at 2 Gy $\left(\mathrm{SF}_{2}\right)$ and $\mathrm{SER}_{0.5} \mathrm{Was}$ illustrated in log scale. Error bars indicated SD. There was an inverse relationship between radiosensitivity and the degree of disulfiram-induced enhancement of radiosensitivity in glioblastoma cells.

\section{Results}

\section{MGMT expression in GBL cell lines}

Western blot results showed that U138MG and T98G cells, but not U251MG, U87MG, or U373MG cells, expressed MGMT (Fig. 1). Thus, U138MG and T98G are MGMT-wt cells while U251MG, U87MG, and U373MG are MGMT-meth cells based on these results, confirming that MGMT methylation is correlated with MGMT protein expression [4]. After treatment of DSF for 24 hours in a concentration of $75 \mathrm{nM}$ for U138MG and $100 \mathrm{nM}$ for others, U138MG and T98G kept the expression of MGMT.

\section{2. $\mathrm{IC}_{50}$ of DSF}

The mean $\mathrm{IC}_{50}$ value \pm standard deviation (SD) was $71.85 \pm$ $0.86 \mathrm{nM}$ for $\mathrm{U} 138 \mathrm{MG}, 101.91 \pm 7.63 \mathrm{nM}$ for T98G, $90.78 \pm 2.22$ $\mathrm{nM}$ for U251MG, 117.65 $\pm 45.76 \mathrm{nM}$ for U87MG, $80.69 \pm 64.40$ $\mathrm{nM}$ for U373MG, and $99.45 \pm 29.43 \mathrm{nM}$ for NHA. From these results, the value for subsequent experiment was determined as $75 \mathrm{nM}$ for U138MG and $100 \mathrm{nM}$ for others.

\section{Enhancement of radiosensitivity with DSF}

Radiosensitivity of GBL cells was enhanced by pre-irradiation treatment with DSF except for U373MG. The enhancement was remarkable in MGMT-wt cells (Fig. 2). However, DSF did not affect radiosensitivity of NHA.

Taking the radiation dose that resulted in surviving fractions (SF) of 0.5 and 0.2 as references in cell survival curve fitting after irradiation alone or irradiation plus DSF, sensitizer enhancement ratios (SERs) were calculated (Fig. 2). The mean $S_{0.5}$ value $\pm S D$ was $1.503 \pm 0.251$ for $U 138 M G, 1.104 \pm$ 0.065 for $\mathrm{T} 98 \mathrm{G}, 1.257 \pm 0.235$ for $\mathrm{U} 251 \mathrm{MG}, 1.121 \pm 0.072$ for U87MG, $0.945 \pm 0.034$ for U373MG, and $0.998 \pm 0.070$ for NHA. The mean $S_{0.2}$ value $\pm S D$ was $1.30 \pm 0.084$ for $U 138 M G$, $1.091 \pm 0.070$ for $\mathrm{T} 98 \mathrm{G}, 1.119 \pm 0.076$ for $\mathrm{U} 251 \mathrm{MG}, 1.10 \pm 0.035$ for U87MG, $0.992 \pm 0.019$ for U373MG, and $0.995 \pm 0.029$ for NHA. The mean $S_{0.5}$ and $S E R_{0.2}$ were $1.192 \pm 0.236$ and $1.121 \pm 0.114$ for all cells, $1.303 \pm 0.273$ and $1.196 \pm 0.134$ for MGMT-wt cells, and $1.148 \pm 0.190$ and $1.087 \pm 0.070$ for MGMT-meth cells, respectively (Fig. 3A).

For comparison of its own radiosensitivity of each GBL cell line, surviving fractions at $2 \mathrm{~Gy}\left(\mathrm{SF}_{2}\right)$ for radiation alone were calculated from cell survival curves. The mean $\mathrm{SF}_{2}$ value $\pm S D$ was $0.884 \pm 0.099$ for U138MG, $0.787 \pm 0.103$ for T98G, $0.826 \pm$ 0.024 for $\mathrm{U} 251 \mathrm{MG}, 0.719 \pm 0.041$ for $\mathrm{U} 87 \mathrm{MG}$, and $0.710 \pm 0.057$ for U373MG. Among these five GBL cell lines, U138MG had 


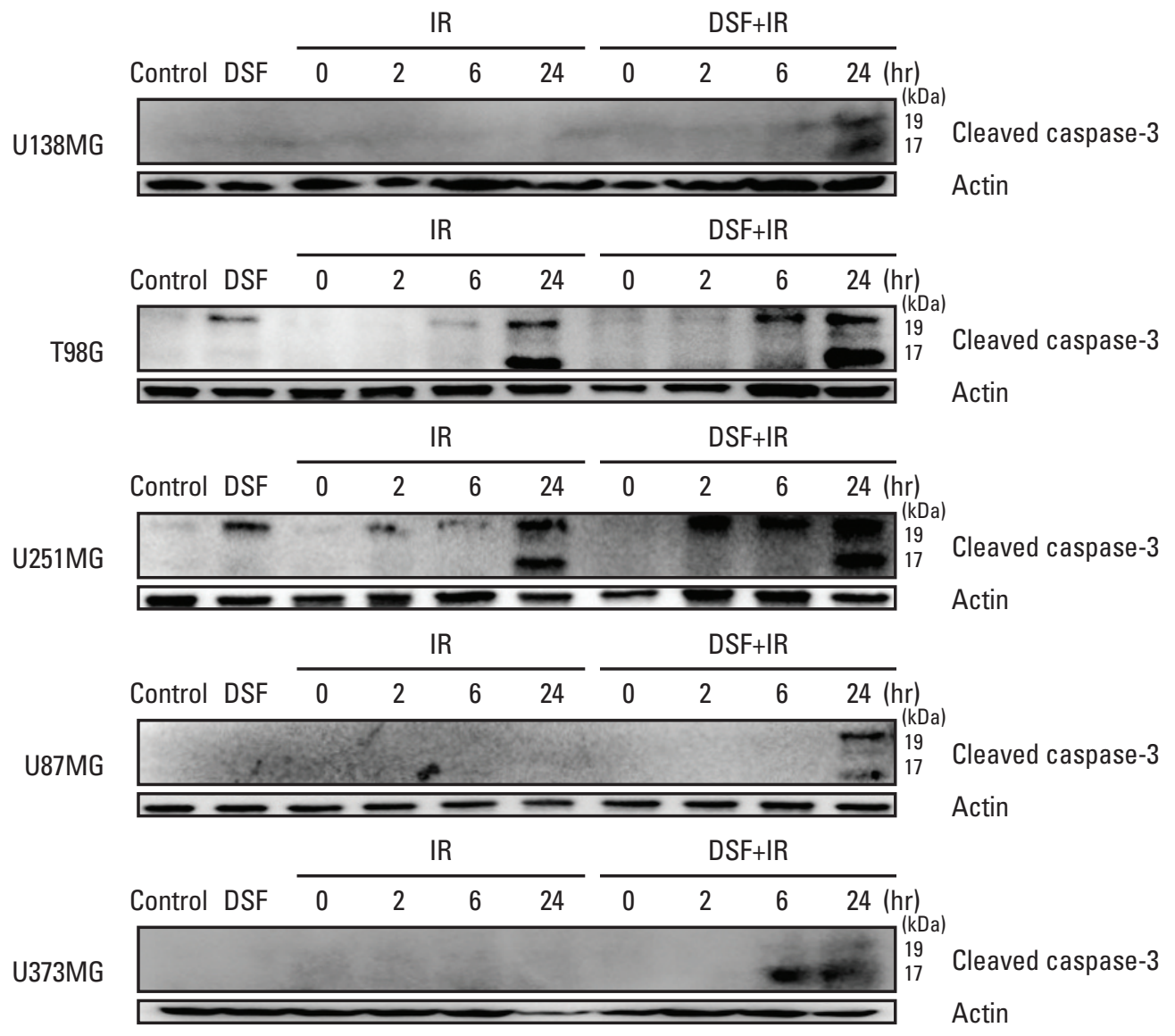

Fig. 4. Western blot analysis of cleaved caspase-3 after 0, 2, 6, and 24 hours of irradiation (IR). Pre-irradiation disulfiram (DSF) either induced expression of cleaved caspase-3 in U138MG, U87MG, and U373MG cells or augmented it in T98G and U251MG cells.

the highest $\mathrm{SF}_{2}$ (i.e., the most radioresistant) while U373MG was the most radiosensitive. When $\mathrm{SER}_{0.5}$ was plotted to $\mathrm{SF}_{2}$, there was an inverse relationship between radiosensitivity and the degree of DSF-induced enhancement of radiosensitivity in GBL cells (Fig. 3B).

\section{Expression of cleaved caspase-3}

In T98G and U251MG cells, cleaved caspase-3 was expressed after DSF treatment alone or at 2 or 6 to 24 hours after irradiation alone. Pre-irradiation DSF strongly augmented its expression after irradiation (Fig. 4).

In U138MG, U87MG, or U373MG cells, cleaved caspase-3 was not expressed after DSF treatment alone or up to 24 hours after irradiation alone. However, it was induced to express by pre-irradiation DSF treatment at 6 and 24 hours after irradiation.

\section{5. $\gamma \mathrm{H} 2 \mathrm{AX}$ assay}

In MGMT-wt cells (U138MG and T98G), $\gamma \mathrm{H} 2 \mathrm{AX}$ foci existed significantly longer by pre-irradiation DSF treatment (Fig. 5A). The proportion of $\gamma \mathrm{H} 2 \mathrm{AX}$ positive cells after irradiation alone was rapidly and continuously decreased up to 24 hours in U138MG and T98G cells. However, it was not decreased till 24 hours in U138MG cells or rather gradually decreased in T98G cells with pre-irradiation DSF. The proportion of $\gamma \mathrm{H} 2 \mathrm{AX}$ positive cells at 24 hours was also significantly higher in both U138MG and T98G cells with preirradiation DSF in compared with irradiation alone (Fig. 5B).

In MGMT-meth cells, the proportion of $\gamma \mathrm{H} 2 \mathrm{AX}$ positive cells after irradiation alone was gradually repaired from 6 hours in U251MG and U87MG cells or sustained till 24 hours in U373MG cells. These patterns were not changed by preirradiation DSF treatment (Fig. 5C). 
A
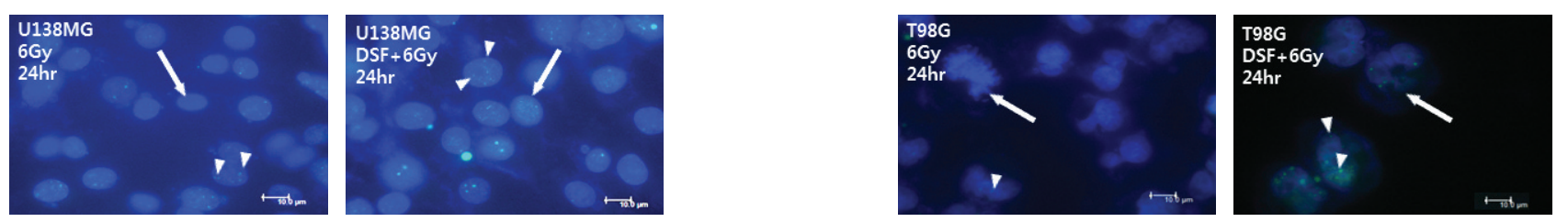

C
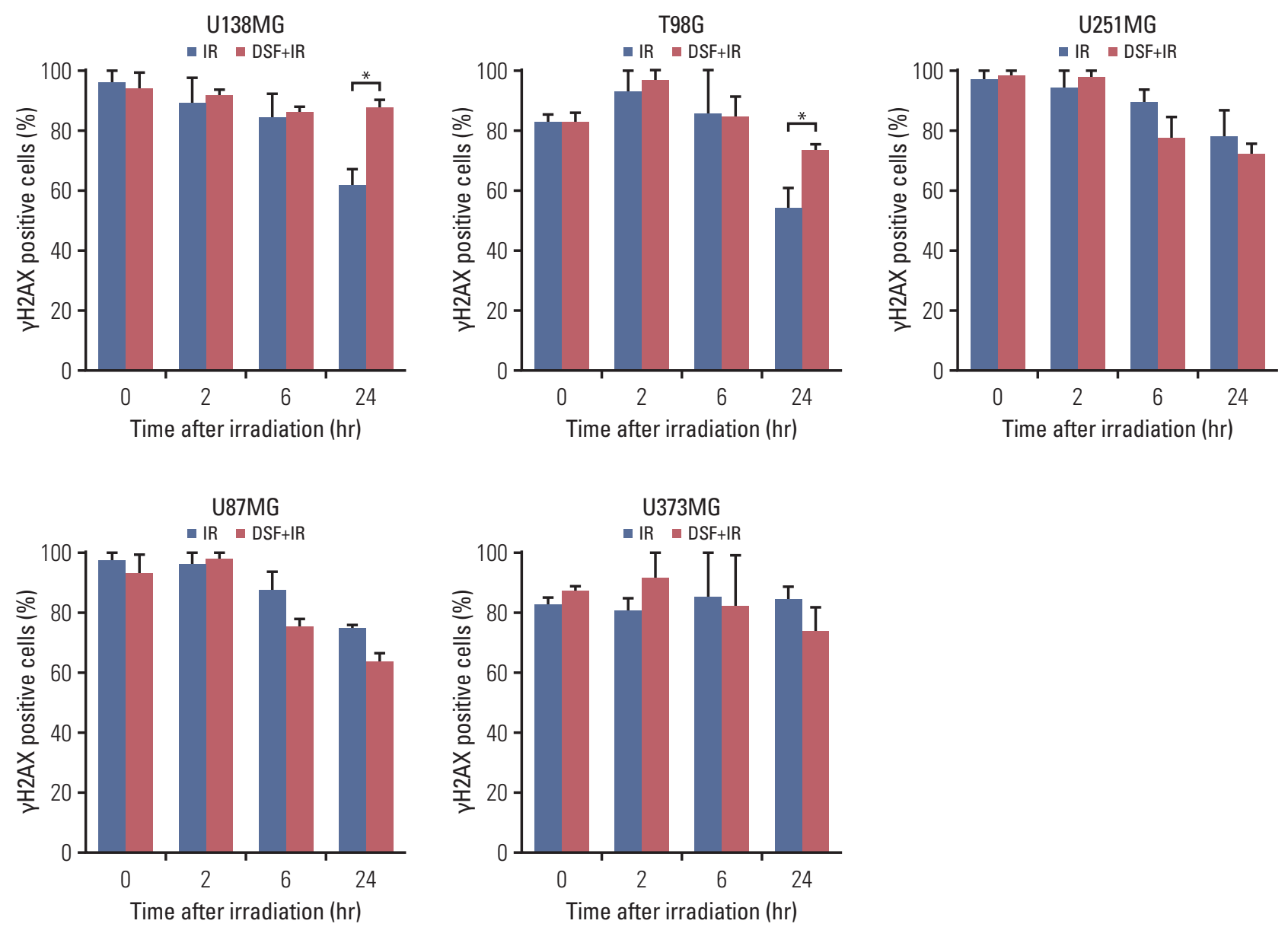

Fig. 5. $\gamma \mathrm{H} 2 \mathrm{AX}$ assay. Anti- $\gamma \mathrm{H} 2 \mathrm{AX}$ antibody was applied and then fluorescein isothiocyanate-labeled secondary antibody was added (arrowheads). Nuclei were counterstained with 4',6-diamidino-2-phenylindole (DAPI, arrows). (A, B) $\gamma \mathrm{H} 2 \mathrm{AX}$ foci after irradiation (IR) were more prevalent in pre-irradiation disulfiram (DSF) treatment group than those in IR (6 Gy) alone group until 24 hours in U138MG and T98G cells with wildtype unmethylated $\mathrm{O}^{6}$-methylguanine-DNA methyltransferase promoter $(\times 1,000)$. Scale bars $=10 \mu \mathrm{m}$. (C) The decrease in proportion of $\gamma \mathrm{H} 2 \mathrm{AX}$ positive cells by the time after IR was significantly blocked by pre-irradiation DSF in U138MG and T98G cells, but was not in U251MG, U87MG, or U373MG cells. ${ }^{*} \mathrm{p}<0.05$. 

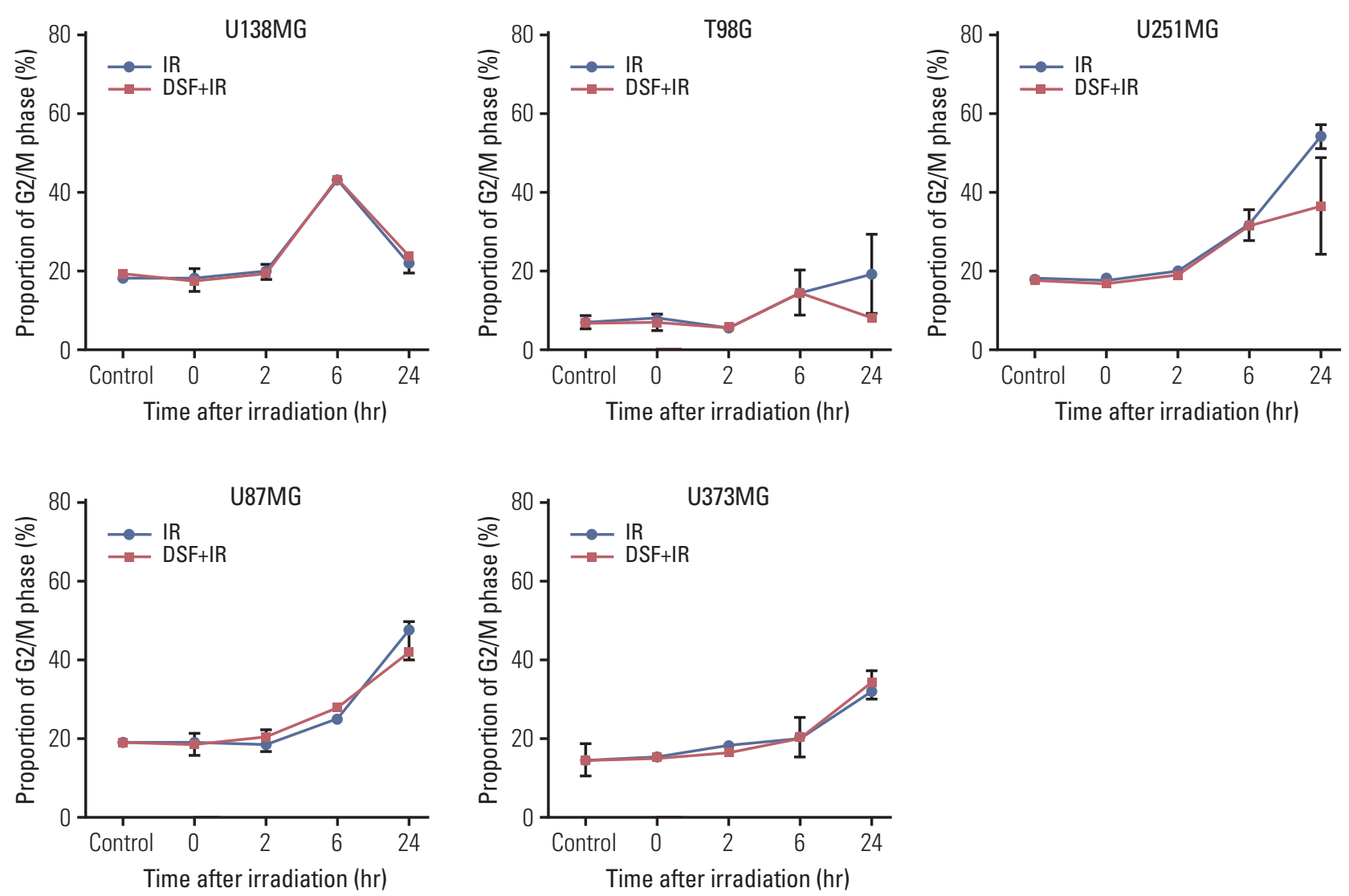

Fig. 6. Proportion of cells at G2/M phase and sub-G1 fraction by flowcytometry. (A) Disulfiram (DSF) abrogated irradiation (IR)-induced G2/M arrest in T98G and U251MG cells. (Continued to the next page)

\section{Cell cycle distribution}

The proportion of cells at G2/M phase was increased at 6 hours after irradiation alone in U138MG cells, but decreased to control level at 24 hours. This kinetic pattern was not influenced by pre-irradiation DSF treatment (Fig. 6A). However, in T98G cells treated with pre-irradiation DSF, the proportion of cells at G2/M phase at 24 hours after irradiation was lower in compared with irradiation alone.

In MGMT-meth cells, kinetic patterns of U251MG cells were very similar to those of T98G cells. However, proportions at each time points were approximately twice of those measured in T98G cells (Fig. 6A). In U87MG and U373MG cells, the proportion of cells at G2/M phase was increased from 6 hours after irradiation alone. This pattern was not influenced by pre-irradiation DSF treatment.

The proportion of sub-G1 fraction at 24 hours after irradiation was increased in compared with that at 0 hour in all cell lines except U373MG (Fig. 6B). These patterns were not affected by pre-irradiation DSF treatment.

\section{Discussion}

This is the first report demonstrating that DSF can induce preferential enhancement of radiosensitivity of GBL cells in compared with normal astrocyte and that DSF-induced radiosensitization is more prominent in cells with radioresistance. These results might have clinical relevance to GBL in general, especially for more resistant GBL with MGMTwt.

GBLs with MGMT-wt have poor prognosis after standard radiochemotherapy. They have little benefit from TMZ [3]. Therefore, there have been two approaches to improve survival of MGMT-wt GBL. One approach is by developing new treatment strategies and the other is by targeting MGMT itself. Enzastaurin, an orally active protein kinase $C$ and phosphoinositide-3 kinase/Akt inhibitor, has been combined with RT in phase II trial. However, it failed to reach the goal of the study [23]. A phase II study that added cilengitide, an integrin inhibitor, to standard radiochemotherapy with TMZ 

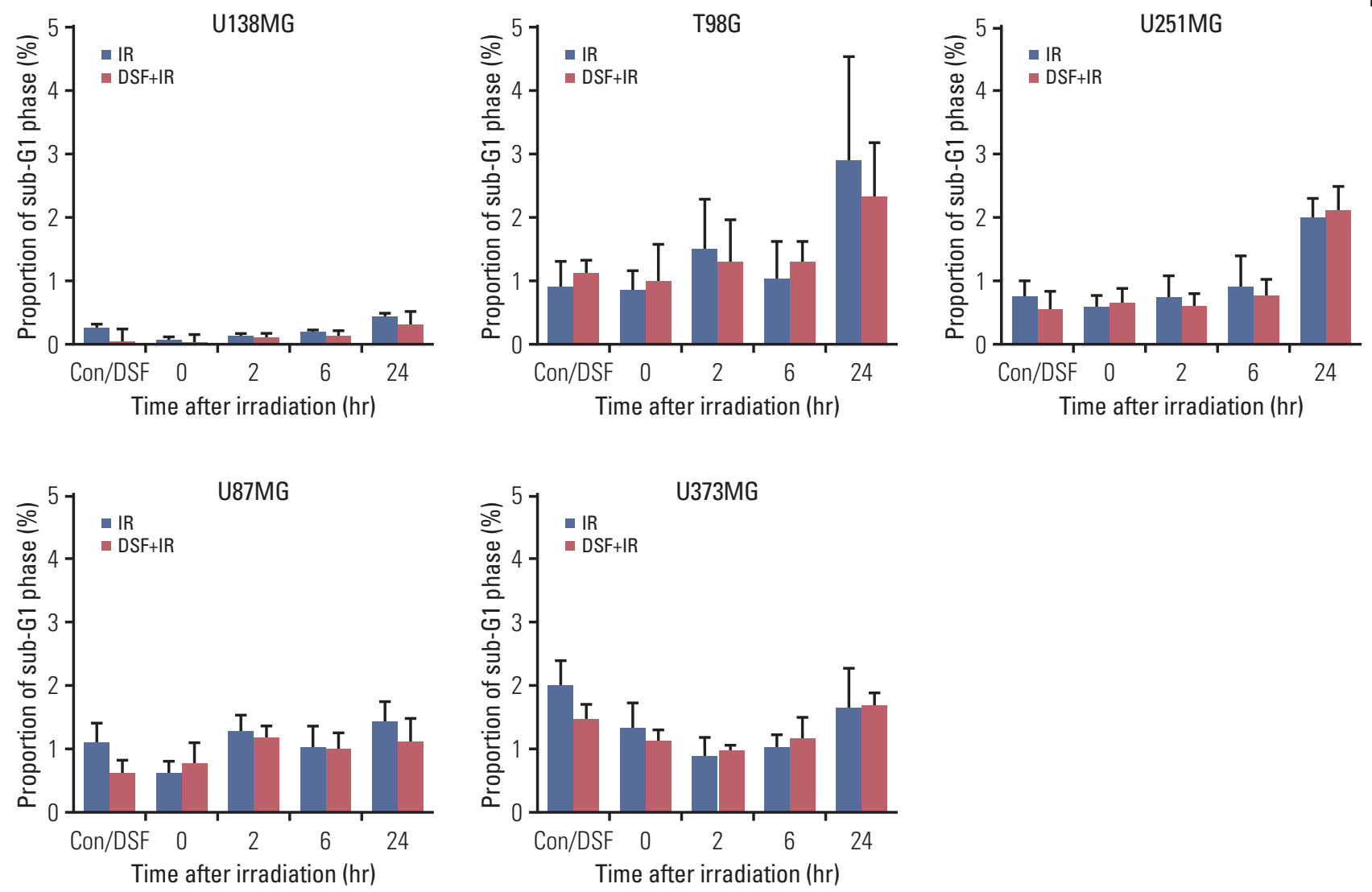

Fig. 6. (Continued from the previous page) (B) DSF failed to affect IR-induced change in sub-G1 fraction. Con, control.

has reported inconclusive outcomes [24]. There has been no dramatic new treatment for MGMT-wt GBL. On the other hand, MGMT itself has emerged as a treatment target [25]. While inhibitors of MGMT as $\mathrm{O}^{6}$-benzylguanine or $\mathrm{O}^{6}$-[4-bromothenyl] guanine have shown positive results in vitro and in animal studies, severe bone marrow toxicities are developed in patients [26].

Synergistic effect of DSF and radiation has been reported in patient-derived AT/RT cells using cell viability assay [11]. It has been further shown that DSF can enhance radiosensitivity of an AT/RT cell line by increasing DNA damage, apoptosis, and autophagy [16]. In a study examining the effect of DSF on sensitivity of neuroblastoma cells to both ionizing radiation and radioisotope, radiosensitization of DSF has been revealed both in vitro and in vivo [17]. However, the mechanism is not reported [17]. Recently, it has been demonstrated that DSF enhances chemoradiation effect in pancreatic cells by especially targeting cancer stem cells both in vitro and in vivo [18].

The current study is the first exploration of possible mechanisms of a radiosensitizing effect of DSF in GBL cells. The current study elucidated that pre-irradiation treatment of DSF increased apoptosis in all GBL cell lines. The effect of DSF on cell cycle distribution was inconclusive. It also delayed DNA damage repair, especially in GBL cells with MGMT-wt. Although there have been previous studies that reported DSF alone acts as a direct and potent inhibitor of MGMT or increases DNA damage possibly by posttranslational regulation $[13,14]$, these effect were not observed in the concentration about $\mathrm{IC}_{50}$ of each cell line in the current study.

DSF has been available for a long time. Its pharmacodynamics and side effects have been well-known. Thus, a phase II trial of DSF as an anti-cancer drug could be accelerated. Costs and time for development of DSF as an anti-cancer drug could be reduced by over $40 \%$ [27]. In addition, DSF is inexpensive because the estimated cost of one year for daily treatment with DSF $500 \mathrm{mg}$ is approximately \$550 [27]. Although the economy of cancer treatment should be considered many factors such as expected survival and complications, simply calculated expected cost of other alternative treatments such as bevacizumab or TTF are much more 
expensive than DFS $[28,29]$. DSF could be very cost effective from development to dissemination as a cancer drug. Recently, in a phase I study, DSF was used for 12 patients during TMZ maintenance after concurrent chemoradiotherapy. Tolerance dose of DSF was $500 \mathrm{mg}$ daily and the median progression-free survival was 8.8 months, which was favorable compared to historical data [30].

Limitation of the current study was that this was just the first step to generalize the effect of DSF to patients with GBL. Further study is required to confirm the role of DSF as a radiosensitizer in GBL in vivo and varied effects according to the methylation status of MGMT promoter of GBL cells.

In summary, DSF has radiosensitizing effect on GBL cells, especially for resistant GBL cells with wildtype unmethylated MGMT promotor, possibly due to increased apoptosis and delayed DNA damage repair. Future studies are needed to confirm these findings in vivo and/or in clinical studies.
Radiotherapy combined with DSF could be a promising strategy for GBL patients, especially for those with wildtype unmethylated MGMT promotor with more resistance to radiotherapy alone or radiochemotherapy with TMZ.

\section{Conflicts of Interest}

Conflict of interest relevant to this article was not reported.

\section{Acknowledgments}

This work was partly supported by Grant No. 03-2017-0080 (20171229) from SNUH Research Fund. We would like to send special thanks to Prof. In Ah Kim at Seoul National University Bundang Hospital, Bundang, Korea and Prof. Sun Ha Paek at Seoul National University Cancer Hospital, Seoul, Korea for kindly providing NHA and U138MG cell lines, respectively.

\section{References}

1. Stupp R, Hegi ME, Mason WP, van den Bent MJ, Taphoorn MJ, Janzer RC, et al. Effects of radiotherapy with concomitant and adjuvant temozolomide versus radiotherapy alone on survival in glioblastoma in a randomised phase III study: 5-year analysis of the EORTC-NCIC trial. Lancet Oncol. 2009;10:45966.

2. Mirimanoff RO, Gorlia T, Mason W, Van den Bent MJ, Kortmann RD, Fisher B, et al. Radiotherapy and temozolomide for newly diagnosed glioblastoma: recursive partitioning analysis of the EORTC 26981/22981-NCIC CE3 phase III randomized trial. J Clin Oncol. 2006;24:2563-9.

3. Hegi ME, Diserens AC, Gorlia T, Hamou MF, de Tribolet N, Weller M, et al. MGMT gene silencing and benefit from temozolomide in glioblastoma. N Engl J Med. 2005;352:997-1003.

4. Costello JF, Futscher BW, Tano K, Graunke DM, Pieper RO. Graded methylation in the promoter and body of the O6-methylguanine DNA methyltransferase (MGMT) gene correlates with MGMT expression in human glioma cells. J Biol Chem. 1994;269:17228-37.

5. Wee CW, Kim E, Kim N, Kim IA, Kim TM, Kim YJ, et al. Novel recursive partitioning analysis classification for newly diagnosed glioblastoma: a multi-institutional study highlighting the MGMT promoter methylation and IDH1 gene mutation status. Radiother Oncol. 2017;123:106-11.

6. Chinot OL, Wick W, Mason W, Henriksson R, Saran F, Nishikawa R, et al. Bevacizumab plus radiotherapy-temozolomide for newly diagnosed glioblastoma. $N$ Engl J Med. 2014;370:709-22

7. Gilbert MR, Dignam JJ, Armstrong TS, Wefel JS, Blumenthal DT, Vogelbaum MA, et al. A randomized trial of bevacizumab for newly diagnosed glioblastoma. N Engl J Med. 2014;370:
699-708.

8. Stupp R, Taillibert S, Kanner AA, Kesari S, Steinberg DM, Toms SA, et al. Maintenance therapy with tumor-treating fields plus temozolomide vs temozolomide alone for glioblastoma: a randomized clinical trial. JAMA. 2015;314:2535-43.

9. Wright C, Moore RD. Disulfiram treatment of alcoholism. Am J Med. 1990;88:647-55.

10. Yang EJ, Wu C, Liu Y, Lv J, Shim JS. Revisiting non-cancer drugs for cancer therapy. Curr Top Med Chem. 2016;16:214455.

11. Choi SA, Choi JW, Wang KC, Phi JH, Lee JY, Park KD, et al. Disulfiram modulates stemness and metabolism of brain tumor initiating cells in atypical teratoid/rhabdoid tumors. Neuro Oncol. 2015;17:810-21.

12. Kast RE, Karpel-Massler G, Halatsch ME. CUSP9* treatment protocol for recurrent glioblastoma: aprepitant, artesunate, auranofin, captopril, celecoxib, disulfiram, itraconazole, ritonavir, sertraline augmenting continuous low dose temozolomide. Oncotarget. 2014;5:8052-82.

13. Paranjpe A, Zhang R, Ali-Osman F, Bobustuc GC, Srivenugopal KS. Disulfiram is a direct and potent inhibitor of human O6-methylguanine-DNA methyltransferase (MGMT) in brain tumor cells and mouse brain and markedly increases the alkylating DNA damage. Carcinogenesis. 2014;35:692-702.

14. Srivenugopal KS, Rawat A, Niture SK, Paranjpe A, Velu C, Venugopal SN, et al. Posttranslational regulation of $\mathrm{O}(6)$ methylguanine-DNA methyltransferase (MGMT) and new opportunities for treatment of brain cancers. Mini Rev Med Chem. 2016;16:455-64.

15. Triscott J, Lee C, Hu K, Fotovati A, Berns R, Pambid M, et al. Disulfiram, a drug widely used to control alcoholism, sup- 
presses the self-renewal of glioblastoma and over-rides resistance to temozolomide. Oncotarget. 2012;3:1112-23.

16. Lee YE, Choi SA, Kwack PA, Kim HJ, Kim IH, Wang KC, et al. Repositioning disulfiram as a radiosensitizer against atypical teratoid/rhabdoid tumor. Neuro Oncol. 2017;19:1079-87.

17. Rae C, Tesson M, Babich JW, Boyd M, Sorensen A, Mairs RJ. The role of copper in disulfiram-induced toxicity and radiosensitization of cancer cells. J Nucl Med. 2013;54:953-60.

18. Cong J, Wang Y, Zhang X, Zhang N, Liu L, Soukup K, et al. A novel chemoradiation targeting stem and nonstem pancreatic cancer cells by repurposing disulfiram. Cancer Lett. 2017;409: 9-19.

19. Kim JH, Moon SH, No M, Kim JJ, Choi EJ, Cho BJ, et al. Isotype-specific inhibition of histone deacetylases: identification of optimal targets for radiosensitization. Cancer Res Treat. 2016;48:1130-40.

20. Fowler JF. The linear-quadratic formula and progress in fractionated radiotherapy. Br J Radiol. 1989;62:679-94.

21. Kim JH, Kim IH, Shin JH, Kim HJ, Kim IA. Sequence-dependent radiosensitization of histone deacetylase inhibitors trichostatin A and SK-7041. Cancer Res Treat. 2013;45:334-42.

22. Kim HJ, Kim JH, Chie EK, Young PD, Kim IA, Kim IH. DNMT (DNA methyltransferase) inhibitors radiosensitize human cancer cells by suppressing DNA repair activity. Radiat Oncol. 2012;7:39.

23. Wick W, Steinbach JP, Platten M, Hartmann C, Wenz F, von Deimling A, et al. Enzastaurin before and concomitant with radiation therapy, followed by enzastaurin maintenance therapy, in patients with newly diagnosed glioblastoma without MGMT promoter hypermethylation. Neuro Oncol. 2013;15: 1405-12.
24. Nabors LB, Fink KL, Mikkelsen T, Grujicic D, Tarnawski R, $\mathrm{Nam} \mathrm{DH}$, et al. Two cilengitide regimens in combination with standard treatment for patients with newly diagnosed glioblastoma and unmethylated MGMT gene promoter: results of the open-label, controlled, randomized phase II CORE study. Neuro Oncol. 2015;17:708-17.

25. Kaina B, Margison GP, Christmann M. Targeting O(6)-methylguanine-DNA methyltransferase with specific inhibitors as a strategy in cancer therapy. Cell Mol Life Sci. 2010;67:3663-81.

26. Blumenthal DT, Rankin C, Stelzer KJ, Spence AM, Sloan AE, Moore DF Jr, et al. A Phase III study of radiation therapy (RT) and $\mathrm{O}(6)$-benzylguanine+BCNU versus $\mathrm{RT}$ and BCNU alone and methylation status in newly diagnosed glioblastoma and gliosarcoma: Southwest Oncology Group (SWOG) study S0001. Int J Clin Oncol. 2015;20:650-8.

27. Cvek B. Nonprofit drugs as the salvation of the world's healthcare systems: the case of Antabuse (disulfiram). Drug Discov Today. 2012;17:409-12.

28. Kovic B, Xie F. Economic evaluation of bevacizumab for the first-line treatment of newly diagnosed glioblastoma multiforme. J Clin Oncol. 2015;33:2296-302.

29. Bernard-Arnoux F, Lamure M, Ducray F, Aulagner G, Honnorat J, Armoiry X. The cost-effectiveness of tumor-treating fields therapy in patients with newly diagnosed glioblastoma. Neuro Oncol. 2016;18:1129-36.

30. Huang J, Campian JL, Gujar AD, Tran DD, Lockhart AC, DeWees TA, et al. A phase I study to repurpose disulfiram in combination with temozolomide to treat newly diagnosed glioblastoma after chemoradiotherapy. J Neurooncol. 2016; 128:259-66. 